\title{
Primary cutaneous Fusarium solani infection in a hemodialysis patient
}

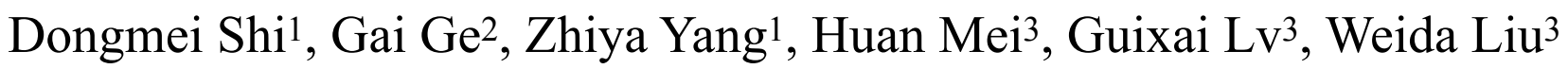

\author{
Authors' affiliations: \\ ${ }^{1}$ Department of Dermatology, Jining No. 1 People's Hospital, Shandong, P. R. China \\ 2 Department of Pathology, Jining No. 2 People's Hospital, Shandong, P. R. China \\ ${ }_{3}^{3}$ Department of Mycology, Institute of Dermatology, Chinese Academy of Medical Sciences \& Peking Union Medical College, Nanjing, \\ Jiangsu, P. R. China
}

\section{Objective:}

Fusarium solani, a hyphomyocetous fungus, is often isolated from the environment as a laboratory contaminant, but it is also known as a pathogen causing keratomycosis, onychomycosis, and opportunistic infection of the skin and viscera.

\section{Method:}

Herein, we describe a case of cutaneous Fusarium solani infection in a patient with chronic kidney failure (uremia), who has a prior history of hemodialysis twice weekly.

\section{Results:}

A 47-year-old Chinese female was admitted to our hospital on 24 November, 2017 (day 0 being the day of hospital admission) for treatment of sustained lesion on her right forearm. About five months ago (at day- 5 months), the patient has a red papule with $0.5 \mathrm{~cm}$ in diameter on her forearm with gentle itching. The lesion gradually becomes larger during the last five months without any treatment. The patient has a history of chronic kidney failure for more than 2 years and has been treated with hemodialysis twice per week. She was given right forearm graft fistula for hemodialysis.

At day 0, the patient was admitted to our hospital with a localized cutaneous erythematous on the right forearm. The erythematous lesion was $20 \mathrm{~cm}$ in diameter with clear edge, which was covered with necrosis and black crust.

Direct microscopic examination of the necrotic area showed numerous fungal elements. Culture on Sabouraud dextrose agar with cycloheximide yielded a floccose, grayish white colony. Microscopically, crescent-shaped macroconidia and oval microconidia were abundant (Figure $2 \mathrm{C}$ and $\mathrm{D}$ ). The fungus was identified using gene analysis as Fusarium solani.

At day 0 , the patient was initially given oral itraconazole 200 mg per day combined with topical ketoconazole and terbinafine cream twice daily. The lesion was improved after one-month treatment.
At day +28 , fungal susceptibilities for each testing antifungals are as follows: itraconazole minimum inhibitory concentration (MIC) is $16 \mu \mathrm{g} / \mathrm{mL}$; terbinafine $\mathrm{MIC},>0.5 \mu \mathrm{g} / \mathrm{mL}$; voriconazole MIC, $16 \mu \mathrm{g} / \mathrm{mL}$; fluconazole, $>64 \mu \mathrm{g} / \mathrm{mL}$; 5 fluorocytosine, $>64 \mu \mathrm{g} / \mathrm{mL}$ and fulvicin $>64 \mu \mathrm{g} / \mathrm{mL}$. According to these MIC data, the patient was treated with terbinafine 0.25 once daily instead of itraconazole in combination of ketoconazole cream topically twice per day. At day +8 weeks, although direct microscopic examination of the scale on the lesion showed that the hypha was thinner than of the first-time examination, there were still full of numerous fungal elements under microscope. Today, the patient is still under our fellow up schedule.

\section{Conclusion:}

We present a first case of cutaneous Fusarium solani infection in a patient with chronic kidney failure. In this case the fungal infection may due to minor trauma by repeated puncture in immunocompromised host. It is believed that the treatment in the early stage of infection prevented further extension of the lesion. The long duration of treatment is needed because of the fungus resistance to most antifungal agents.

Keywords: Fusarium solani; hemodialysis; chronic kidney failure

Figure 2
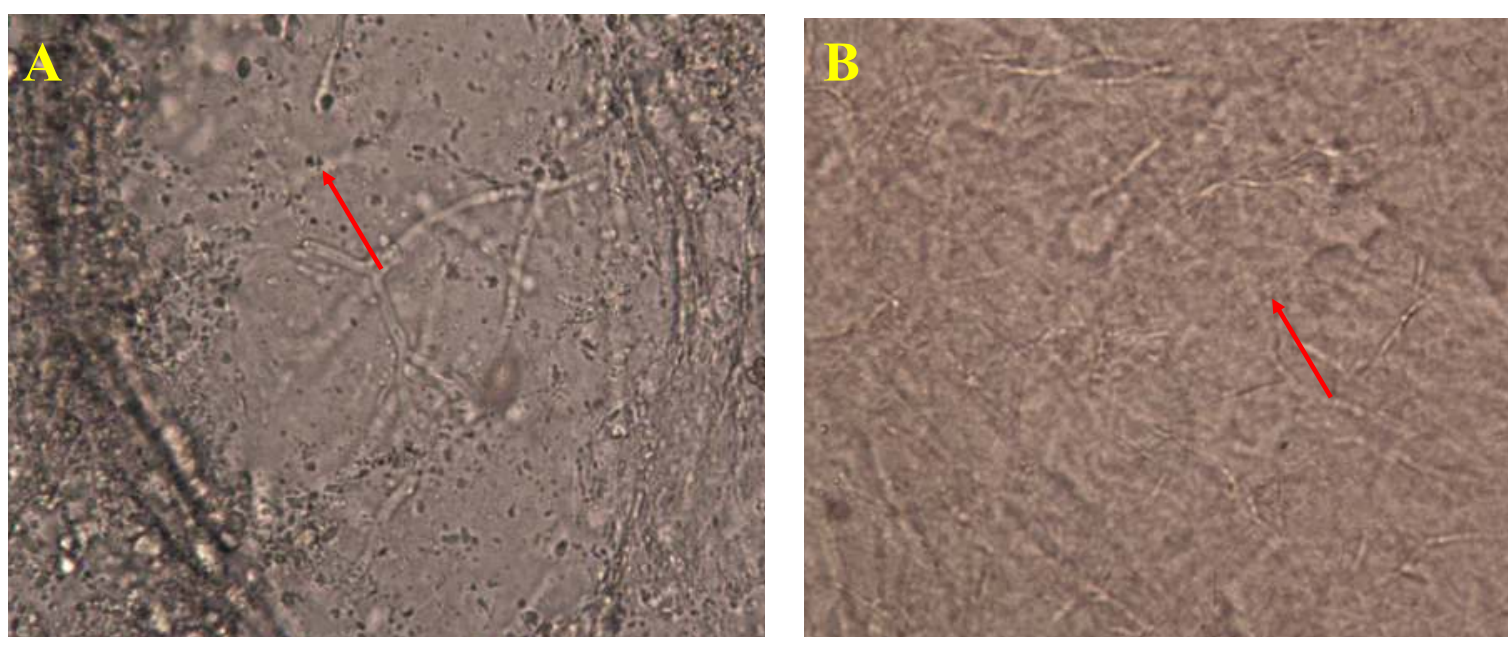

Direct examination of $10 \%$ potassium hydroxide wet mounts of the scales of the lesions revealed abundant light, branched and septatehyphae(original magnificent, $400 \times)$. A: At day 0 and B: At day +28 .

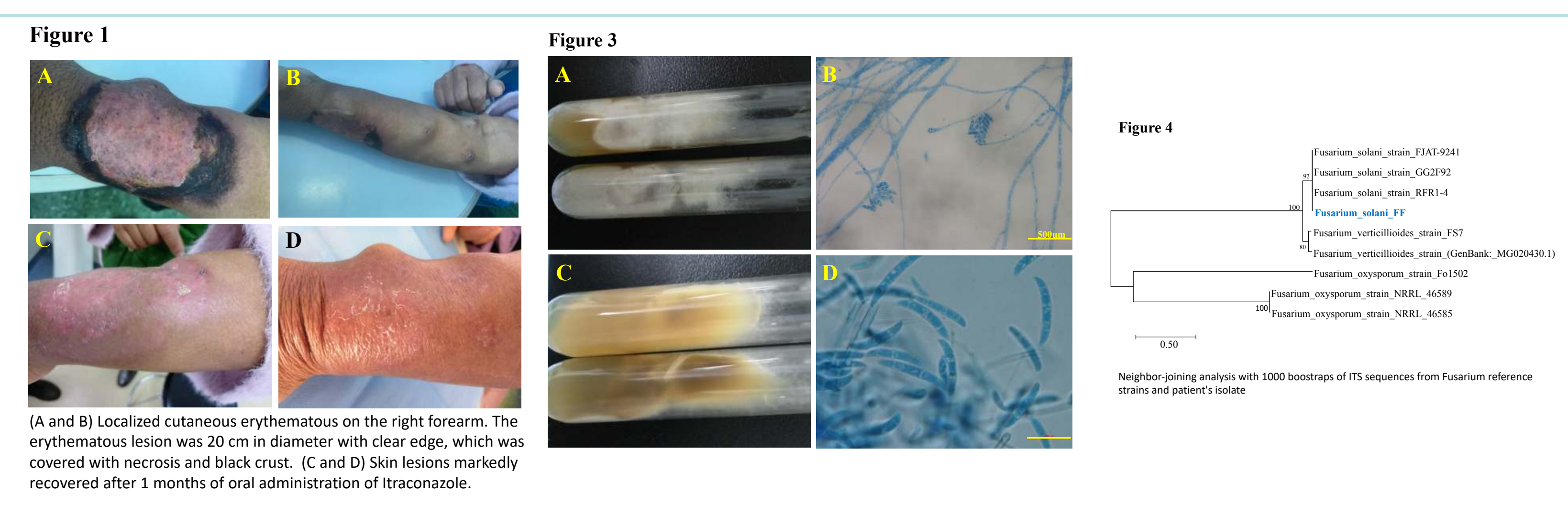

